$1-2002$

\title{
Ultrafast Flow Quantification With Segmented K-Space Magnetic Resonance Phase Velocity Mapping
}

\author{
Haosen Zhang \\ Cleveland State University \\ Sandra S. Halliburton \\ Cleveland State University \\ James R. Moore \\ Siemens Medical Solutions
}

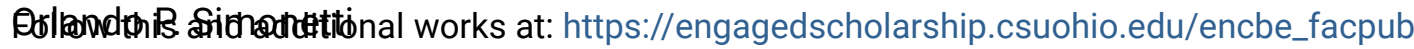

Si-mens Medical Solutions

Part of the Biochemical and Biomolecular Engineering Commons, and the Biomedical Engineering and

BaudogR.eSahyartzmams

Glevelabd Clinice Foundatipn work benefit you? Let us know!

\section{Publisher's Statement}

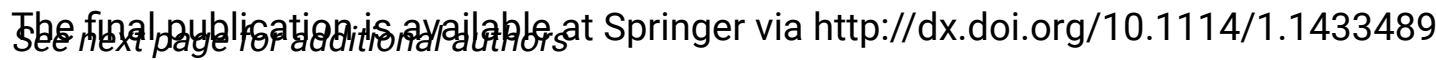

\section{Original Citation}

Zhang, H., Halliburton, S. S., Moore, J. R., Simonetti, O. P., Schvartzman, P. R., White, R. D., , \& Chatzimavroudis, G. P. (2002). Ultrafast Flow Quantification With Segmented k-Space Magnetic Resonance Phase Velocity Mapping. Annals of Biomedical Engineering, 30(1), 120-128.

\section{Repository Citation}

Zhang, Haosen; Halliburton, Sandra S.; Moore, James R.; Simonetti, Orlando P.; Schvartzman, Paulo R.; White, Richard D.; and Chatzimavroudis, George P., "Ultrafast Flow Quantification With Segmented K-Space Magnetic Resonance Phase Velocity Mapping" (2002). Chemical \& Biomedical Engineering Faculty Publications. 76. https://engagedscholarship.csuohio.edu/encbe_facpub/76

This Article is brought to you for free and open access by the Chemical \& Biomedical Engineering Department at EngagedScholarship@CSU. It has been accepted for inclusion in Chemical \& Biomedical Engineering Faculty Publications by an authorized administrator of EngagedScholarship@CSU. For more information, please contact library.es@csuohio.edu. 


\section{Authors}

Haosen Zhang, Sandra S. Halliburton, James R. Moore, Orlando P. Simonetti, Paulo R. Schvartzman, Richard D. White, and George P. Chatzimavroudis 


\title{
Ultrafast Flow Quantification With Segmented $k$-Space Magnetic Resonance Phase Velocity Mapping
}

\author{
HaOSEn Zhang, ${ }^{1,2}$ SANdra S. Halliburton, ${ }^{2}$ JAmes R. MOORE, ${ }^{3}$ Orlando P. Simonetti, ${ }^{3}$ \\ Paulo R. SchVARTZMan, ${ }^{2}$ Richard D. White, ${ }^{2}$ and George P. Chatzimavroudis ${ }^{1,2}$ \\ ${ }^{1}$ Laboratory of Biofluid Mechanics and Cardiovascular Imaging, Department of Chemical Engineering, Cleveland State University, \\ Cleveland, $\mathrm{OH} ;{ }^{2}$ Section of Cardiovascular Imaging, Division of Radiology, The Cleveland Clinic Foundation, Cleveland, \\ $\mathrm{OH}$; and ${ }^{3}$ Magnetic Resonance Division, Siemens Medical Systems, Chicago, IL
}

\section{INTRODUCTION}

Magnetic resonance (MR) phase-velocity mapping $(\mathrm{PVM})$ is a technique that has been widely used clinically to measure blood flow. The technique provides traditional anatomical information using MR imaging and blood velocity information in any spatial direction using

the phase-velocity encoding technique. This technique is based on the fact that, by using the proper magnetic-field gradients, the velocity can be encoded within the phase of the detected signal. This phase-velocity encoding follows a linear equation:

$$
\phi=\gamma \nu M_{1},
$$

where $\phi$ is the phase of the received signal (rad), $\gamma$ is the gyromagnetic ratio $(\mathrm{Hz} / \mathrm{T}), \nu$ is the velocity (assumed constant) $(\mathrm{m} / \mathrm{s})$, and $M_{1}$ is the first moment of the gradient wave form $\left(\mathrm{T} \mathrm{s}^{2} / \mathrm{m}\right)$ at the echo time (TE):

$$
M_{1}=\int_{0}^{\mathrm{TE}} G(t) t d t
$$

where $G(t)$ is the magnetic-field gradient (T/m). Two acquisitions are performed, one in which the velocity is compensated and one in which the velocity is encoded. By subtracting the two images pixel by pixel with respect to the phase of the signal, a phase image can be constructed which contains velocity information for each pixel according to Eq. (1).

Modern PVM was invented in 1982 by Moran. ${ }^{24}$ There have been many in vitro and clinical studies evaluating the potential and reliability of PVM for flow characterization or quantification. In vitro, the accuracy of PVM was found to be consistently high, with errors of less than $10 \% .^{4-6,8,12,21}$ In vivo studies showed a good correlation between PVM and conventional velocimetric (Doppler ultrasound) and flowmetric techniques. ${ }^{4,13,21,22,26,27}$ The clinical application of PVM to quantify or visualize blood flow in the heart, through heart valves, and in the great vessels has been extensive. . $^{3,16-18,22,26,30}$ With the development of more powerful hardware and software, flow quantification in 
TABLE 1. Flow conditions.

\begin{tabular}{cccc}
\hline Tube No. & Tube ID $(\mathrm{cm})$ & $\begin{array}{c}\text { Steady flow rate } \\
(\mathrm{ml} / \mathrm{s})\end{array}$ & $\begin{array}{c}\text { Pulsatile flow volume } \\
(\mathrm{ml} / \mathrm{cycle})\end{array}$ \\
\hline 1 & 0.56 & $1.7,5,8.3,16.7$ & 6 \\
2 & 1.47 & $6.7,16.7,66.7,116.7$ & 20 and 30 \\
3 & 2.02 & $10,25,33.3,83.3,166.7$ & 40 and 60 \\
4 & 2.62 & $16.7,50,116.7,200$ & 70 and 90 \\
\hline
\end{tabular}

smaller vessels, such as the coronary arteries, has become possible.

Conventional PVM involves a gradient-echo sequence with bipolar velocity-encoding gradients applied in the desired direction for velocity measurement. The acquisition can be prospectively or retrospectively referenced to the ECG signal of the subject in order to acquire a number of velocity measurements (time phases) throughout the cardiac cycle. Only one line of $k$-space (frequency domain) is acquired for each phase per heartbeat. As a result, the acquisition of a single velocity component requires several minutes (typically, 3-5 min, depending on functional and imaging parameters). Considering that blood flow measurement is usually only part of a complete cardiac MR examination and that new velocimetric diagnostic approaches have started to involve multislice and multidirectional velocity acquisitions, $8,31,32$ this single $k$-space line (nonsegmented) acquisition technique becomes less practical clinically.

With the development of rapid imaging sequences, it has become possible to implement PVM much faster. The two most prominent sequences for ultrafast PVM are multishot echo-planar imaging (EPI) and turbo gradient echo (TGE). Instead of acquiring a single $k$-space line during each time phase per cardiac cycle, multiple lines or segments of $k$-space lines are acquired during each time phase. Consequently, the acquisition can be performed in seconds, instead of minutes.

The similarity between segmented $k$-space TGE and multishot EPI is that both acquire multiple lines or segments of $k$-space per phase, in contrast to the conventional, nonseqmented, gradient-echo sequence that acquires only a single line. The difference is that in TGE acquisition of each line of the segment requires a separate excitation, whereas in EPI all lines of the segment are acquired after a single excitation. Because of the absence of multiple excitation pulses, EPI is generally faster. However, the longer effective echo time of EPI combined with the specific gradient function of EPI cause flow-related signal voids, especially in relatively disturbed flow fields, and other image artifacts. TGE has shown better flow behavior and is, therefore, more promising for reliable ultrafast flow quantification with relatively disturbed flow and significant vessel motion (e.g., coronary arteries). ${ }^{9,10,14,20,25}$ In addition, previous in vitro and clinical studies ${ }^{2,10,11,14,19,20,23,25,28,29}$ evaluating both
EPI and TGE techniques for PVM have indicated that TGE is generally superior in terms of temporal and $\left(\right.$ sub-millimeter ${ }^{28}$ ) in-plane spatial resolution.

The aim of this study was to evaluate the segmented $k$-space TGE sequence in quantifying flow from throughplane velocity measurements. An in vitro study was performed under a variety of flow conditions and imaging parameters, followed by preliminary clinical measurements.

\section{METHODS}

In vitro and in vivo studies were performed in a $1.5 \mathrm{~T}$ Siemens Sonata whole-body MRI scanner (Siemens Medical Systems, Erlangen, Germany) with a maximum gradient strength of $40 \mathrm{mT} / \mathrm{m}$. First, in vitro PVM measurements were performed with steady and pulsatile water flow through four straight PVC tubes with inside diameters of $5.6 \mathrm{~mm}$ (tube No. 1), $14.7 \mathrm{~mm}$ (tube No. 2), $20.2 \mathrm{~mm}$ (tube No. 3), and $26.2 \mathrm{~mm}$ (tube No. 4). The tubes were placed in a water container to insure detection of adequate MR signal. Then, in vivo PVM measurements were performed in the ascending aorta of two human subjects, one with an ascending aortic aneurysm, and one with ischemic heart disease.

\section{Experimental Conditions}

Steady flow studies were first performed using a range of flow rates $(1.7-200 \mathrm{ml} / \mathrm{s}$, Table 1). The Reynolds number (Re) range studied was 400-10,500. The true flow rate was known via precalibrated rotameters. Then, pulsatile flow studies (see the flow loop in Fig. 1) were

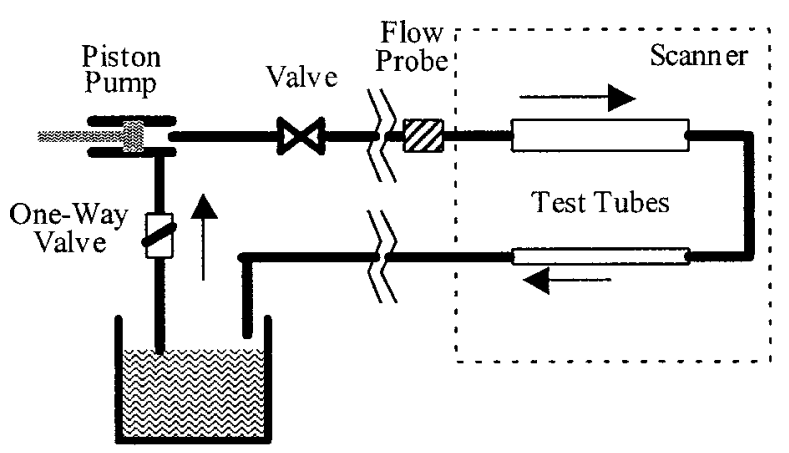

FIGURE 1. Pulsatile flow loop. 


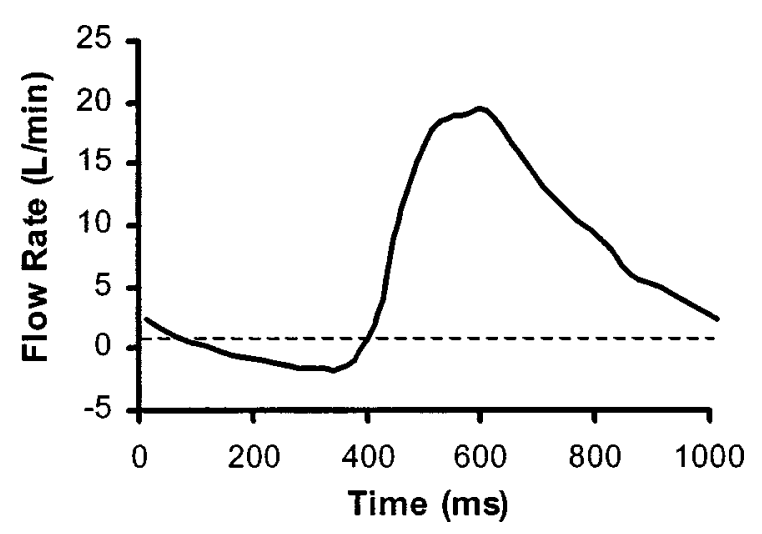

FIGURE 2. True flow wave form generated by the piston pump as measured by the MR-compatible ultrasonic flow probe.

conducted using a computer-controlled piston pump (SuperPump, SPS 3891, Vivitro Systems Inc., Victoria, British Columbia, Canada) to provide flow pulsatility. A hardware/software system (Vivigen Waveform Generator VG8991, Vivitro Systems Inc.) was used to program and download a sinusoidal flow wave form from a PC to the piston pump. The piston stroke volumes studied were $6-90 \mathrm{ml} /$ cycle (Table 1) under a rate of $60 \mathrm{cycles} / \mathrm{min}$. The true flow wave form (Fig. 2) was measured with a precalibrated, MR-compatible (brass), transit-time ultrasonic flow probe ( $20 \mathrm{~N}$ in-line, Transonic Systems, Inc., Ithaca, NY). The flow data from the probe was acquired by a single channel flow meter (T-106, Transonic Systems, Inc.). The flow wave form was recorded on a PC by digitizing the analog signal from the flow meter with an analog-to-digital board (PCI-MIO-16E-4, National Instruments Inc., Austin, TX), using the LabVIEW software (Version 5.0, National Instruments, Inc.). Integration of the flow curve during the cycle provided the true flow volume.

\section{In Vitro Imaging Procedure}

The test section (water-filled container with submerged straight tubes) was connected to the flow loop, and the entire system was inserted into the bore of the scanner with the test section placed at the isocenter. Initial true fast imaging with steady-state precession (FISP) scout images in all three spatial orientations (axial, coronal, and sagittal) showed the location of the tubes. An imaging slice was then placed axially, perpendicular to the long axis of the tube under study. Three PVM acquisitions of the through-plane velocity were performed for each flow condition, using the following three sequences: (a) conventional nonsegmented gradient echo with one $k$-space line per time phase and cycle; (b) segmented TGE with five $k$-space lines per time phase and cycle; and (c) segmented TGE with nine lines per time phase and cycle. In all sequences, the flow-sensitive and the flow-compensated data (which were subtracted to provide the phase data) were acquired in an interleaved manner in the same cycle. The flip angle was $30^{\circ}$. The slice thickness (ST) was 5 or $3 \mathrm{~mm}$. The field of view (FOV) was $160 \times 160 \mathrm{~mm}^{2}$ (for tube No. 1), $200 \times 200$ $\mathrm{mm}^{2}$ (for tube Nos. 1, 2, and 3), and $300 \times 300 \mathrm{~mm}^{2}$ (for tube Nos. 2, 3, and 4). The acquisition matrix size was $192 \times 256$ for sequence (a), $140 \times 256$ for sequence (b), and $144 \times 256$ for sequence (c). The nominal voxel size varied between $0.6 \times 0.8 \times 3$ and $0.6 \times 1.6 \times 5 \mathrm{~mm}^{3}$ for sequence (a), between $0.6 \times 1.1 \times 3$ and $0.6 \times 2.1 \times 5 \mathrm{~mm}^{3}$ for sequence (b), and between $0.6 \times 1.1 \times 3$ and 0.6 $\times 2.1 \times 5 \mathrm{~mm}^{3}$ for sequence (c). Therefore, for a reconstructed matrix of $256 \times 256$, the voxel size (after interpolation) varied from $0.6 \times 0.6 \times 3$ to $1.2 \times 1.2 \times 5 \mathrm{~mm}^{3}$ in all sequences. The shortest possible TE $(2.3-3.5 \mathrm{~ms})$ was used. In sequence (a), the repetition time (TR) was $30 \mathrm{~ms}$. In sequences (b) and (c), the time to acquire data for any one $k_{y}$ line (actual TR) varied between 5.2 and $5.6 \mathrm{~ms}$, depending on the imaging parameters, such as ST and FOV. The echo was acquired asymmetrically within the data acquisition period. The peak of the echo occurred at data-point 82 of 256 and the missing data points were zero filled. The velocity encoding value (above which aliasing would take place) was 20-150 $\mathrm{cm} / \mathrm{s}$, depending on the magnitude of the flow.

In the case of pulsatile flow, a transistor-transistor logic (TTL) signal synchronized with the piston pump flow wave form triggered the scanner to acquire multiple time phases throughout the cycle. The procedure was similar to the standard clinical situation in which the ECG signal from the subject is used to trigger the scanner for data acqusition. Using echo view sharing (adjacent time phases shared some of the acquired $k$-space lines), the temporal resolution was further improved in the segmented sequences [30 ms for sequence (a), 30-45 $\mathrm{ms}$ for sequence (b), and 50-65 ms for sequence (c)]. The time corresponding to each phase was the time of acquisition of the central line of the segment, since that line contained the most useful velocity information of the segment. The number of time phases during the $1 \mathrm{~s}$ cycle was 31 for sequence (a), 22-31 for sequence (b), and 15-20 for sequence (c). The scanning duration was 3.2 $\mathrm{min}$ for sequence (a), $28 \mathrm{~s}$ for sequence (b), and $16 \mathrm{~s}$ for sequence (c).

\section{Clinical Measurements}

To evaluate the clinical potential of segmented $k$-space PVM in quantifying blood flow, preliminary measurements were performed in two human subjects (subject No. 1: male, 70 years old, ascending aortic aneurysm; subject No. 2: male, 60 years old, ischemic heart disease). Velocity data were acquired with a slice positioned in the ascending aorta of each subject, at the level 
of the pulmonary artery bifurcation. Two sequences were used: (i) the conventional nonsegmented gradient echo; and (ii) the segmented TGE with nine lines per segment. ECG gating was used to acquire multiple time phases throughout the cardiac cycle. The sequence with five lines per segment used in the in vitro measurements was not tested in the in vivo part, because of its long duration $(\geqslant 25 \mathrm{~s}$; more difficult to be performed within a breath hold). The ST was $5 \mathrm{~mm}$ for both subjects. The scan percentages were $75 \%$ and $56 \%$ for sequences (i) and (ii), respectively. The FOV was $263 \times 350 \mathrm{~mm}^{2}$ for subject No. 1 (rectangular FOV factor of $75 \%$ ), and 225 $\times 300 \mathrm{~mm}^{2}$ for subject No. 2 (rectangular FOV factor of $75 \%$ ). Combination of the scan percentage with the rectangular FOV factor resulted in acquired numbers of 144 and $108 k_{y}$ lines for sequence (i) and (ii), respectively. The velocity encoding value was $250 \mathrm{~cm} / \mathrm{s}$, and the rest of the imaging parameters were as in the in vitro part. The scan durations for the first sequence were 2.4 and 2.1 min for subject Nos. 1 and 2, respectively. The second sequence was performed within a breath hold (12 and $11 \mathrm{~s}$ for subject Nos. 1 and 2, respectively). For subject No. 1, the numbers of time phases acquired were 31 for the nonsegmented sequence and 19 for the nineline segmented sequence. For subject No. 2, these numbers were 25 and 15 , respectively.

\section{Data Analysis}

Each PVM acquisition produced a magnitude and a phase image (or a series of magnitude and phase images during the cycle under pulsatile flow and in the clinical cases). All images were transferred to a work station (Ultra-10, SUN Microsystems, Inc., Palo Alto, CA). A computer program was used to convert the phase values of the phase images to velocity values $(\mathrm{cm} / \mathrm{s})$, based on Eq. (1). Then, the images were visualized using TRANSFORM (Version 3.4, Research Systems, Inc., Boulder, $\mathrm{CO}$ ). The tube (in vitro) or the aortic (in vivo) cross section was clearly visualized and selected, and the fluid velocity was integrated over the tube/aortic crosssectional area to find the flow rate $(\mathrm{ml} / \mathrm{s})$. For the pulsatile flow in vitro data and for the clinical flow data, integration of the flow curve over the cycle provided the flow volume ( $\mathrm{ml} /$ cycle).

Regression analysis, nonparametric statistical tests (sign test), ${ }^{15}$ and Bland-Altman analysis ${ }^{1}$ were performed to compare the PVM-measured flow values with the true flow values (in vitro) and the flow data from the segmented techniques with the flow data from the nonsegmented technique (in vitro and in vivo). The MINITAB software (Version 13, Minitab, Inc., State College, PA) was used for the statistical analysis. A $p$ value $<0.05$ demonstrated a significant difference.
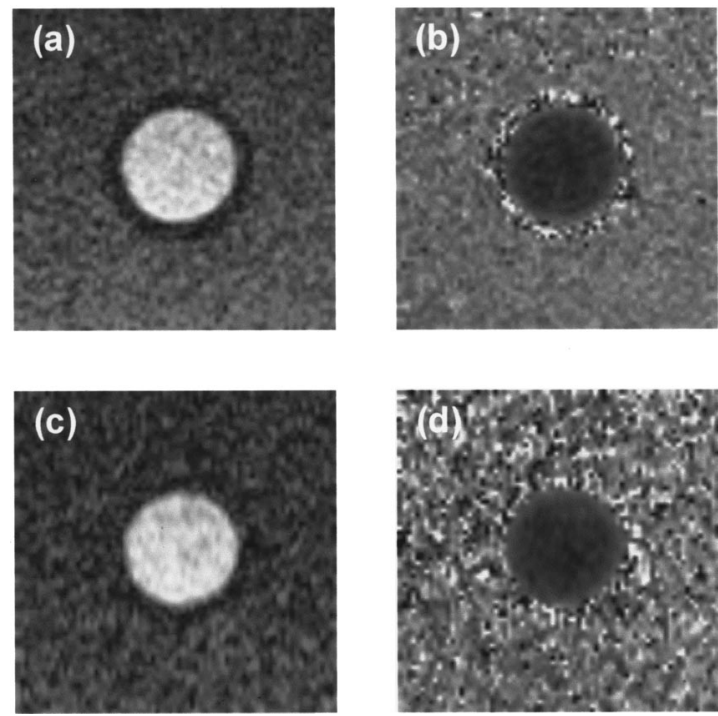

FIGURE 3. Magnitude (a) and phase (b) images acquired using the nonsegmented PVM sequence. Magnitude (c) and phase (d) images using the segmented phase velocity mapping (PVM) sequence with nine lines per segment. The phase images contain [encoded according to Eq. (1)] the velocity information.

\section{RESULTS}

Figure 3 shows the magnitude and phase images from nonsegmented and nine-line segmented PVM acquisitions in the in vitro case. Both images from the nonsegmented [Figs. 3(a) and 3(b)] and the segmented [Figs. $3(\mathrm{c})$ and 3(d)] sequences were of sufficient quality for analysis and flow quantification.

Figure 4 shows the measured velocity profiles in the case of laminar $(\operatorname{Re}=580)$, and non-laminar $(\mathrm{Re}=5250)$ steady flow for the nonsegmented acquisition and the segmented with nine lines of $k$-space per segment. The velocity was normalized with respect to the crosssectional average velocity. The velocity profiles are very similar in the nonsegmented and the segmented cases for
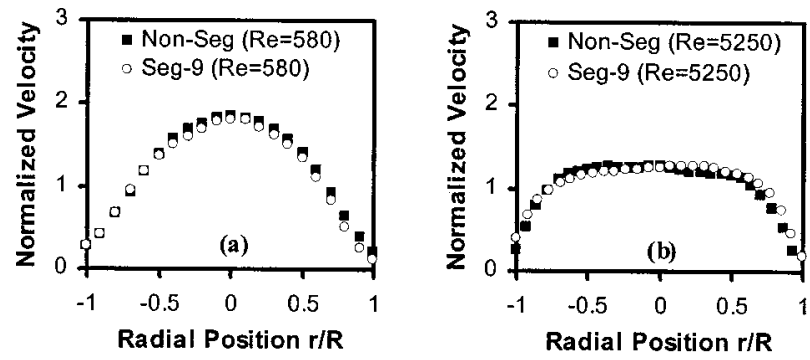

FIGURE 4. Normalized velocity profiles as measured using the nonsegmented (slow) sequence and the segmented (ultrafast) sequence with nine $k$-space lines per segment. Normalization was performed by dividing the local velocity values with the average cross-sectional velocity. (a) Laminar flow; (b) nonlaminar flow. 
TABLE 2. Regression analysis and sign test results for the steady flow data.

\begin{tabular}{lccccc}
\hline $\mathbf{Y}(\mathrm{ml} / \mathrm{s})$ & $\mathbf{X}(\mathrm{ml} / \mathrm{s})$ & Regression equation & $R^{2}$ & $\mathrm{SE}^{\mathrm{a}}(\mathrm{ml} / \mathrm{s})$ & Sign test $p$ value \\
\hline Seg $^{\mathrm{b}}-5$ & True & $Y=1.04 X-0.61$ & 0.990 & 6.2 & 0.33 \\
Seg-9 $^{\text {True }}$ & $Y=1.05 X-0.77$ & 0.989 & 6.5 & 0.89 \\
Seg-5 & Non-Seg & $Y=1.01 X-0.44$ & 0.998 & 3.1 & 0.33 \\
Seg-9 & Non-Seg & $Y=1.02 X-0.63$ & 0.998 & 3.0 & 0.49 \\
\hline
\end{tabular}

aSE: standard error.

'Seg: segmented $k$-space sequence.

'Non-Seg: Nonsegmented k-space sequence.

both flow regimes. In laminar flow, the profile is parabolic, whereas in nonlaminar flow, the profile is flatter. The ratio of the cross-sectional average velocity to the maximum measured velocity was found to be approximately 0.55 for $\mathrm{Re}=580$ and 0.80 for $\mathrm{Re}=5250$. The velocity gradient approaching the wall is greater in nonlaminar flow than in laminar flow.

The known accuracy of the nonsegmented sequence $^{4-6,8,12,21}$ was confirmed in this study $(Y=1.03$ $X-0.04, R^{2}=0.988$, standard error $=6.7 \mathrm{ml} / \mathrm{s} ; Y$ : measured flow rate, $X$ : true flow rate). Close agreement between the measured flow rate from the two segmented sequences and the true flow (from the rotameters), as well as between the flow rate from the two segmented (ultrafast) sequences and the flow rate from the nonsegmented (slow) sequence was found with regression analysis (Table 2). The agreement was also confirmed by sign tests which produced $p$ values much greater than 0.05 in all cases.

The accuracy of the flow rate measurements under nonlaminar flow conditions was found to be comparable to that of the laminar flow. The mean errors [ (measuredtrue)/true] for laminar flow were $1.6 \%, 1.6 \%$, and $0.1 \%$ for the nonsegmented, five-line segmented, and nine-line segmented sequences, respectively; for the nonlaminar case, the errors were $5.1 \%, 3.6 \%$, and $4.5 \%$, respectively. Sign tests confirmed the high accuracy of the measured
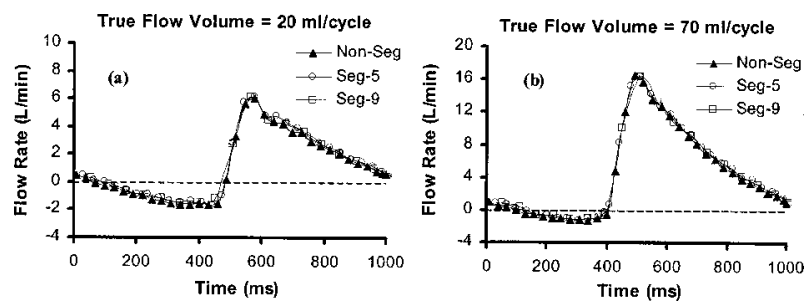

FIGURE 5. Measured flow wave forms under pulsatile flow conditions. (a) True flow volume $=20 \mathrm{ml} /$ cycle; measured flow volume $=17.9,20.8$, and $20.2 \mathrm{ml} /$ cycle for the nonsegmented, the five-line segmented and the nine-line segmented sequences, respectively. (b) True flow volume $=70 \mathrm{ml} /$ cycle; measured flow volume $=71.7,76.0$, and $75.0 \mathrm{ml} / \mathrm{cycle}$ for the nonsegmented, the five-line segmented and the nine-line segmented sequences, respectively. flow rates showing comparable $p$ values $(\gg 0.05)$ for both the laminar and nonlaminar flow cases.

Figure 5 shows the measured flow wave forms for true flow volumes of 20 and $70 \mathrm{ml} / \mathrm{cycle}$ in the pulsatile flow studies. The measured flow curves for the different sequences are very similar both qualitatively and quantitatively. Figure 6 shows the comparison between the PVM-measured flow volumes with all three sequences (the nonsegmented and the two segmented) and the true flow volume known via the flow probe. The mean errors were $3.3 \%, 0.1 \%$, and $0.5 \%$ for the nonsegmented, fiveline segmented, and nine-line segmented sequences, respectively. Table 3 shows the regression analysis results between the three sequences and the flow probe (true) values as well as between the two segmented (fast) sequences and the nonsegmented (slow) one. As in the steady flow case, the results show a very good agreement between the measured and the true flow rate values for all sequences, and a very good agreement between the segmented and the nonsegmented sequences. These find-

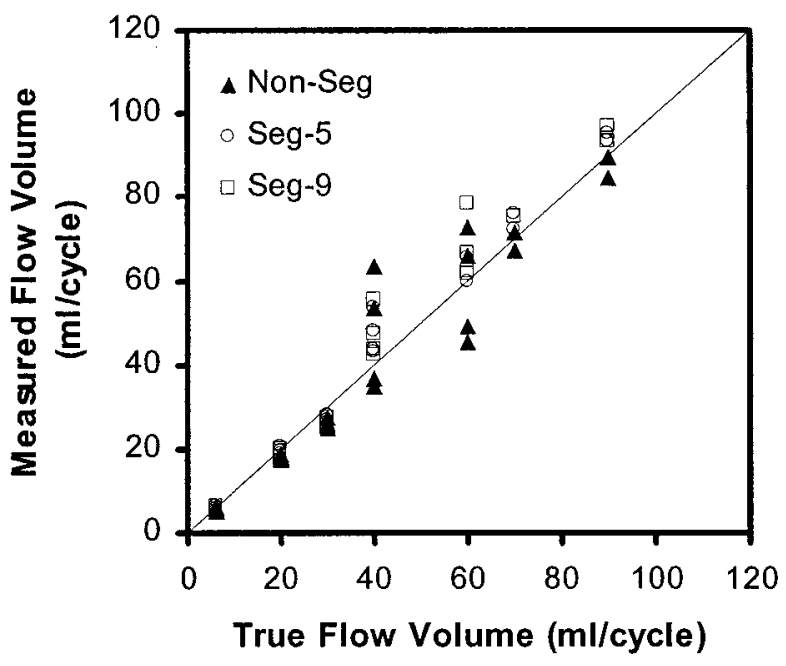

FIGURE 6. Comparison between the PVM-measured and the true flow volumes under pulsatile flow conditions for all sequences used (Non-Seg: nonsegmented acquisition; Seg-5: segmented acquisition with five $k$-space lines per segment; and Seg-9: segmented acquisition with nine $k$-space lines per segment). 
TABLE 3. Regression analysis and sign test results for the pulsatile flow data.

\begin{tabular}{cccccc}
\hline $\begin{array}{c}\mathbf{Y} \\
\text { (ml/cycle) }\end{array}$ & $\begin{array}{c}X \\
(\mathrm{ml} / \text { cycle })\end{array}$ & Regression equation & $R^{2}$ & $\begin{array}{c}\mathrm{SE}^{\mathrm{a}} \\
(\mathrm{ml} / \text { cycle })\end{array}$ & Sign test $p$ value \\
\hline Seg $^{\mathrm{b}}-5$ & True & $Y=1.08 X-1.65$ & 0.983 & 3.7 & 0.84 \\
Seg-9 & True & $Y=1.12 X-2.52$ & 0.971 & 5.1 & 0.84 \\
Seg-5 & Non-Seg & $Y=1.02 X+1.21$ & 0.954 & 6.1 & 0.13 \\
Seg-9 & Non-Seg & $Y=1.07 X+0.10$ & 0.958 & 6.1 & 0.17 \\
\hline
\end{tabular}

${ }^{\text {aSE: }}$ standard error.

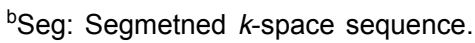

'Non-Seg: Nonsegmetned $k$-space sequence.

ings were confirmed by sign tests (Table 3), in which all $p$ values are greater than 0.05 . In addition, Fig. 7 shows the Bland-Altman analysis plot for the comparison between the measured flow volumes with the nine-line segmented sequence and the true flow volumes. The mean and the standard deviation are low (approximately 0.3 and $2.9 \mathrm{ml} /$ cycle, respectively), and the data points are within the " \pm 2 standard deviations" lines. Similar analysis was performed to compare the flow volumes from the other sequences with the true flow volumes, and the results were identical to the ones presented for the nineline segmented sequence.

Under both steady and pulsatile flow conditions, slice thickness and field of view did not seem to affect the accuracy of the results. Sign tests showed no significant differences $(p \gg 0.05)$ between the two STs ( 3 vs $5 \mathrm{~mm}$ ) and the different FOVs.

The aortic flow measurements in the two human subjects showed a close agreement between the nonsegmented and the nine-line segmented sequences. The flow wave forms were almost identical (Fig. 8). Integration of the flow curves over the entire cardiac cycle showed agreement (difference $\approx 3 \%$ ) in the calculated flow volumes between the nonsegmented sequence and the nine-

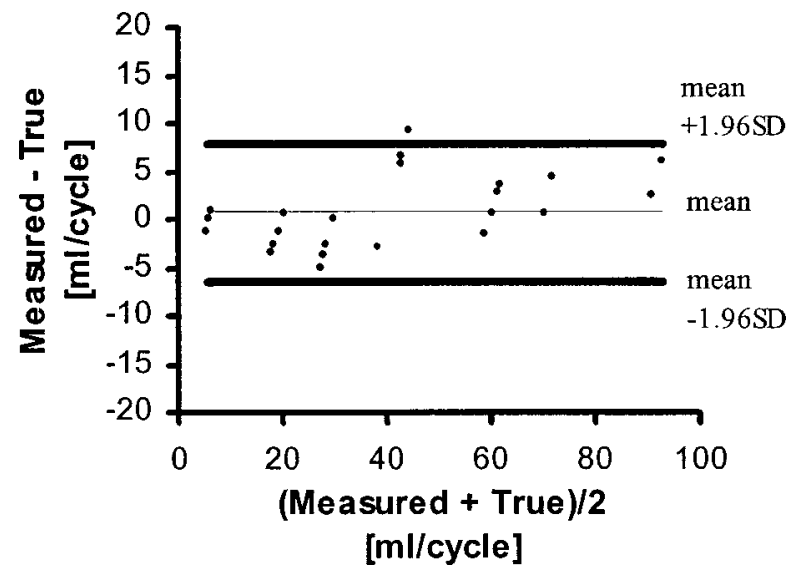

FIGURE 7. Bland-Altman analysis plot comparing the measured and true flow volumes for the nine-line segmented sequence. line segmented sequence (subject No. 1, nonsegmented: $88.1 \mathrm{ml} /$ beat, segmented: $86.1 \mathrm{ml} /$ beat; subject No. 2, nonsegmented: $54.6 \mathrm{ml} /$ beat, segmented: $56.4 \mathrm{ml} /$ beat).

\section{DISCUSSION}

Magnetic resonance phase velocity mapping is a reliable velocimetric and flow quantification technique. It has been clinically used to measure the flow rate and analyze the flow patterns in the heart and vessels such as the entire aorta, the carotid arteries, the renal arteries, cerebral and peripheral vessels, and small vessels such as the coronary arteries. The accuracy and reliability of PVM has been shown through a number of in vitro and in vivo studies in the past. Nevertheless, conventional nonsegmented PVM is relatively slow. It takes $3-5$ min for a single measurement. Fast techniques are necessary to make PVM more practical clinically. This study attempted to evaluate two segmented PVM acquisition schemes, one with five $k$-space lines per segment and one with nine lines per segment, and compare the flow

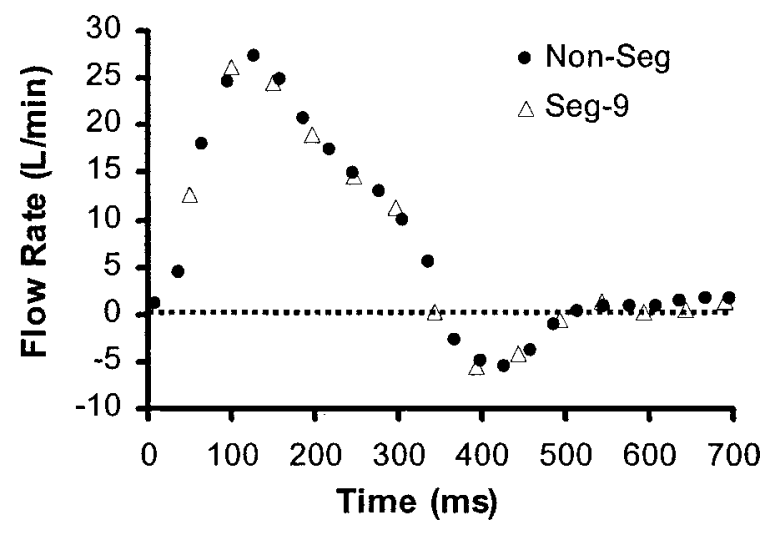

FIGURE 8. Measured flow wave forms in the ascending aorta (level of pulmonary artery bifurcation) of a patient with an ascending aortic aneurysm using the nonsegmented sequence and the nine-line segmented sequence (Non-Seg: nonsegmented acquisition; Seg-9: segmented acquisition with nine $k$-space lines per segment). 
results to those acquired with the nonsegmented sequence as well as with true flow values. PVM measurements under steady and pulsatile flow conditions showed that both segmented sequences provided equally accurate and reliable results. Increasing the number of $k$-space lines to nine did not show any negative effect on the measured flow.

Image quality was very good for all sequences used (Fig. 3) and for all flow conditions facilitating image analysis and processing. Under laminar flow conditions, the flow profiles were parabolic and smooth [Fig. 4(a)]. The centerline velocity was almost twice as large as the average cross-sectional velocity. Under nonlaminar flow conditions, the profiles were flatter and the maximum velocity was much less than twice the cross-sectional average velocity [Fig. 4(b)]. The velocity gradient towards the wall was greater in nonlaminar flow than in laminar flow. This agreement of the measured velocity profiles with what is theoretically and empirically expected (parabolic profiles for laminar flow, flat-like profiles for nonlaminar flow) reflects the velocimetric reliability of the MR PVM technique. Although the in-plane spatial resolution was adequate to obtain the details of the velocity profile, an even higher resolution would better resolve the velocity gradient immediately adjacent to the tube wall. This would be important to calculate wall shear stress. It should be noted that, technically, it was possible to increase spatial resolution by further decreasing the voxel size. However, the effects of reducing voxel size on the signal-to-noise ratio and thus image quality need to be thoroughly investigated by taking into account that there is always a distance between optimal in vitro resolution and optimal in vivo resolution. In addition, improving the resolution means lengthening the acquisition, which is undesired clinically. This limitation at the wall did not affect the quantification of flow rate, because of the lower velocity magnitudes near the wall and the small contribution of those pixels to the overall flow rate.

Of interest is the high accuracy (errors $\leqslant 5 \%$ ) of the flow rate results under nonlaminar flow conditions. Traditionally, MR imaging has shown limitations in measuring the velocity in regions with turbulence, because of voxel signal loss due to velocity fluctuations. Signal loss automatically means loss of the phase (velocity) information. The technological advancement in MR hardware has led to improved magnetic-field gradient performance, which in combination with decreased echo times (the time between tissue excitation and signal readout) can reduce the effects of turbulence on the voxel signal quality, thus allowing velocity measurements under disturbed flow conditions. This study used very small TEs (as low as $2.3 \mathrm{~ms}$ ), because of the ability to operate the very strong magnetic-field gradients very fast. As a result, PVM acquisition provided clear images in which the signal was not reduced. This led to accurate velocity information, which, in turn, resulted in accurate flow rate quantification.

It should be emphasized, however, that the results of this study do not show that turbulent flow is universally quantifiable by PVM. There are many fluid mechanics and technical factors involved that need investigation to draw safe conclusions on this matter. This study merely showed that under the studied range of Re (400-10,500), flow can be measured very accurately by ultrafast PVM sequences. Further investigations are necessary, under larger $\mathrm{Re}$ to show the relationship between decreasing TE and the ability to measure turbulent flow with PVM.

The pulsatile flow results (flow volumes) showed similar levels of accuracy (errors $<5 \%$ ) as the steady flow results. The measured flow curves were very similar in all PVM sequences used (Fig. 5). A similar agreement was observed in the two in vivo cases examined. It should be noted that, in segmented PVM with $n$ lines per segment (in which $n$ is an odd number), the most useful information is contained in the central line, $(n+1) / 2$, of the segment, because this is the closest line of the segment to the center of $k$-space. Therefore, the time corresponding to any phase acquired during the cycle was adjusted to be the time of acquisition of the central $k$-space line of the segment. Without such an adjustment, a temporal shift of the flow curves would take place, as previously observed. ${ }^{28}$ This shift would increase with the number of lines per segment. Combination of this temporal adjustment with retrospective ECG gating is necessary clinically to avoid loss of information in the very beginning and the very end of the cycle.

The close agreement of the calculated flow rates and flow volumes with the true flow values, as well as the agreement of the velocity profiles, flow rates, flow volumes, and flow wave forms between the sequences, suggest that accurate flow quantification is possible using ultrafast segmented PVM. The high in vitro accuracy of the faster nine-line sequence, in combination with its good temporal resolution ( $>10$ phases per cycle) and shorter duration $(<20 \mathrm{~s})$ compared to the five-line sequence, makes it preferable, considering the difficulty some patients have with longer breath holds. This is further supported by the preliminary clinical agreement found between the nonsegmented and the nine-line segmented flow results in the two subjects examined in this study. A larger clinical study will provide more information about the clinical feasibility, advantages, and problems of segmented PVM techniques.

The in vitro part of this study evaluated two segmented PVM sequences using straight rigid tubes under a variety of flow conditions and imaging parameters. Flow conditions (range, pulsatility) and tube size partially simulated what is observed clinically, but no other physiologic factors, such as wall compliance, wall mo- 
tion, presence of stenosis, tube curvature, branching, etc., were studied. The aim of this study was to focus on the sequences and evaluate them under relatively simple flow environments without involving other factors which would complicate the situation. Nevertheless, further evaluation of these sequences under more physiological environments and conditions, and using all three spatial velocity components, is necessary in order to further increase their reliability and broaden their clinical applicability.

Increasing the number of $k$-space lines per segment above nine will certainly shorten the acquisition even more, but at the expense of temporal resolution. Therefore, the number of lines should be increased only after assuring acquisition of an adequate number of phases per cycle in order to prevent loss of valuable information, particularly at peak systole. In addition, it is recommended that, before clinically implementing a sequence, data from in vitro or in vivo evaluation studies be available to assure reliability.

\section{CONCLUSIONS}

Steady and pulsatile flow experiments in straight rigid tubes showed that $k$-space segmented PVM provides accurate, ultrafast velocity measurements and flow quantification. The technique quantified laminar and nonlaminar flow up to a Reynolds number of 10,500 with reliability. Increasing the number of $k$-space lines per segment to nine did not show any negative effects on the ability of PVM to measure flow with high accuracy. Preliminary clinical findings agreed with the experimental findings. As interest in obtaining detailed quantitative information about blood flow noninvasively is continuously increasing, fast protocols are in demand. Ultra-fast segmented $k$-space MR phase velocity mapping shows promise to clinically satisfy this need.

\section{ACKNOWLEDGMENTS}

The authors would like to thank David Epperly for his skillful work and help in the construction of the experimental setup. Support by Siemens Medical Systems is greatly appreciated.

\section{NOMENCLATURE}

$\begin{array}{ll}\text { MR } & \text { magnetic resonance } \\ \text { PVM } & \text { phase velocity mapping } \\ \text { EPI } & \text { echo planar imaging } \\ \text { TGE } & \text { turbo gradient echo } \\ \text { TE } & \text { echo time } \\ \text { TR } & \text { repetition time } \\ \text { ST } & \text { slice thickness } \\ \text { FOV } & \text { field of view } \\ \text { VENC } & \text { velocity encoding limit value }\end{array}$

Re Reynolds number

SE standard error

FISP fast imaging with steady-state precession

TTL transistor-transistor logic

\section{REFERENCES}

${ }^{1}$ Bland, J. M., and D. G. Altman. Statistical methods for assessing agreement between two methods of clinical measurements. Lancet 1:307-310, 1986.

${ }^{2}$ Bock, M., S. O. Schoenberg, L. R. Schad, M. V. Knopp, M. Essig, and G. van Kaick. Interleaved gradient-echo planar (IGEPI) and phase contrast CINE-PC flow measurements in the renal artery. J. Magn. Reson. Imaging 8:889-895, 1998.

${ }^{3}$ Bogren, H. G., and M. H. Buonocore. Blood flow measurements in the aorta and major arteries with MR velocity mapping. J. Magn. Reson. Imaging 4:119-130, 1994.

${ }^{4}$ Bryant, D. J., J. A. Payne, D. N. Firmin, and D. B. Longmore. Measurement of flow with NMR imaging using a gradient pulse and phase difference technique. J. Comput. Assist. Tomogr. 8:588-593, 1984.

${ }^{5}$ Chatzimavroudis, G. P., P. G. Walker, J. N. Oshinski, R. H. Franch, R. I. Pettigrew, and A. P. Yoganathan. Slice location dependence of aortic regurgitation measurements with MR phase velocity mapping. Magn. Reson. Med. 37:545-551, 1997.

${ }^{6}$ Chatzimavroudis, G. P., P. G. Walker, J. N. Oshinski, R. H. Franch, R. I. Pettigrew, and A. P. Yoganathan. The importance of slice location on the accuracy of aortic regurgitation measurements with magnetic resonance phase velocity mapping: An in vitro investigation. Ann. Biomed. Eng. 25:644652, 1997.

${ }^{7}$ Chatzimavroudis, G. P., J. N. Oshinski, R. H. Franch, R. I. Pettigrew, P. G. Walker, and A. P. Yoganathan. Quantification of aortic regurgitation with magnetic resonance phase velocity mapping: A clinical investigation of the importance of slice location. J. Heart Valve Disease 7:94-101, 1998.

${ }^{8}$ Chatzimavroudis, G. P., J. N. Oshinski, R. I. Pettigrew, P. G. Walker, R. H. Franch, and A. P. Yoganathan. Quantification of mitral regurgitation with magnetic resonance phase velocity mapping using a control volume method. J. Magn. Reson. Imaging 8:577-582, 1998.

${ }^{9}$ Chuang, M. L., M. H. Chen, V. C. Khasgiwala, M. V. McConnell, R. R. Edelman, and W. J. Manning. Adaptive correction of imaging plane position in segmented $k$-space cine cardiac MRI. J. Magn. Reson. Imaging 7:811-814, 1997.

${ }^{10}$ Davis, C. P., P.-F. Liu, M. Hauser, S. C. Göhde, G. K. von Schulthess, and J. F. Debatin. Coronary flow and coronary flow reserve measurements in humans with breath-held magnetic resonance phase contrast velocity mapping. Magn. Reson. Med. 37:537-544, 1997.

${ }^{11}$ Debatin, J. F., D. A. Leung, S. Wildermuth, R. Botnar, J. Felblinger, and G. C. McKinnon. Flow quantitation with echo-planar phase-contrast velocity mapping: In vitro and in vivo evaluation. J. Magn. Reson. Imaging 5:656-662, 1995.

${ }^{12}$ Duerk, J. L. and P. M. Pattanu. In-plane flow velocity quantification along the phase encoding axis in MRI. Magn. Reson. Imaging 6:321-333, 1988 .

${ }^{13}$ Dulce, M.-C., G. H. Mostbeck, M. O'Sullivan, M. Cheitlin, G. R. Caputo, and C. B. Higgins. Severity of aortic regurgitation: Interstudy reproducibility of measurements with velocity-encoded cine MR imaging. Radiology 185:234-240, 1992.

${ }^{14}$ Firmin, D. N., R. H. Klipstein, G. L. Hounsfield, M. P. Paley, and D. B. Longmore. Echo-planar high-resolution flow ve- 
locity mapping. Magn. Reson. Med. 12:316-327, 1989.

${ }^{15}$ Hines, W. W., and D. C. Montgomery. Probability and Statistics in Engineering and Management Science, 3rd Ed., New York: Wiley, 1990, p. 732.

${ }^{16}$ Kilner, P. J., G. Z. Yang, R. H. Mohiaddin, D. N. Firmin, and D. B. Longmore. Helical and retrograde secondary flow patterns in the aortic arch studied by three-directional magnetic resonance velocity mapping. Circulation 88:2235-2247, 1993.

${ }^{17}$ Klipstein, R. H., D. N. Firmin, S. R. Underwood, R. S. O. Rees, and D. B. Longmore. Blood flow patterns in the human aorta studied by magnetic resonance, Br. Heart J. 58:316323, 1987.

${ }^{18}$ Kondo, C., G. R. Caputo, R. Semelka, E. Foster, A. Shimakawa, and C. B. Higgins. Right and left ventricular stroke volume measurements with velocity-encoded cine MR imaging: In vitro and in vivo validation. Am. J. Roentgenol. 157:9-16, 1991.

${ }^{19}$ Laffon, E., R. Lecesne, V. de Ledinghen, N. Valli, P. Couzigou, F. Laurent, J. Drouillard, D. Ducassou, and J.-L. Barat. Segmented 5 versus nonsegmented flow quantitation comparison of portal vein flow measurements. Invest. Radiol. 34:176-180, 1999.

${ }^{20}$ McKinnon, G. C., J. F. Debatin, D. R. Wetter, and G. K. von Schulthess. Interleaved echo planar flow quantitation. Magn. Reson. Med. 32:263-267, 1994.

${ }^{21}$ Meier, D., S. Maier, and P. Bosiger. Quantitative flow measurements on phantoms and on blood vessels with MR. Magn. Reson. Med. 8:25-34, 1988.

${ }^{22}$ Mohiaddin, R. H., S. L. Wann, R. Underwood, D. N. Firmin, S. Rees, and D. B. Longmore. Vena caval flow; assessment with cine MR velocity mapping. Radiology 177:537-541, 1990 .

${ }^{23}$ Mohiaddin, R. H., P. D. Gatehouse, and D. N. Firmin. Exercise-related changes in aortic flow measured with spiral echo-planar MR velocity mapping. J. Magn. Reson. Imaging 5:159-163, 1995.
${ }^{24}$ Moran, P. R. A flow velocity zeugmatographic interlace for NMR imaging in humans. Magn. Reson. Imaging 1:197-203, 1982.

${ }^{25}$ Nagel, E., A. Bornstedt, J. Hug, B. Schnackenburg, E. Wellnhofer, and E. Fleck. Noninvasive determination of coronary blood flow with magnetic resonance imaging: Comparison of breath-hold and navigator techniques with intravascular ultrasound. Magn. Reson. Med. 41:544-549, 1999.

${ }^{26}$ Pelc, L. R., N. J. Pelc, S. C. Rayhill, L. J. Castro, G. H. Glover, R. J. Herfkens, D. C. Miller, and R. B. Jeffrey. Arterial and venous blood flow: Noninvasive quantification with MR imaging. Radiology 185:809-812, 1992.

${ }^{27}$ Pettigrew, R. I., W. Dannels, J. R. Galloway, T. Pearson, W. Millikan, J. M. Henderson, J. Peterson, and M. E. Bernardino. Quantitative phase-flow MR imaging in dogs by using standard sequences: Comparison with in vivo flow-meter measurements. Am. J. Roentgenol. 148:411-414, 1987.

${ }^{28}$ Poutanen, V.-P., R. Kivisaari, A.-M. Häkkinen, S. Savolainen, P. Hekali, and C.-G. Standertskjöld-Nordenstam. Multiphase segmented $k$-space velocity mapping in pulsatile flow wave forms. Magn. Reson. Imaging 16:261-270, 1998.

${ }^{29}$ Thomsen, C., M. Cortsen, L. Söndergaard, O. Henriksen, F. Ståhlberg. A segmented $k$-space velocity mapping protocol for quantification of renal artery blood flow during breathholding. J. Magn. Reson. Imaging 5:393-401, 1995.

${ }^{30}$ Underwood, S. R., D. N. Firmin, R. H. Klipstein, R. S. O. Rees, and D. B. Longmore. Magnetic resonance velocity mapping: Clinical application of a new technique. Br. Heart J. 57:404-412, 1987.

${ }^{31}$ Walker, P. G., S. Oyre, E. M. Pedersen, K. Houlind, F. S. A. Guenet, and A. P. Yoganathan. A new control volume method for calculating valvular regurgitation. Circulation 92:579586, 1995.

${ }^{32}$ Walker, P. G., K. Houlind, C. Djurhuus, W. Y. Kim, and E. M. Pedersen. Motion correction for the quantification of mitral regurgitation using the control volume method. Magn. Reson. Med. 43:726-733, 2000. 